\title{
High-energy femtosecond photonic crystal fiber laser
}

Article in Optics Letters · October 2010

DOI: 10.1364/OL.35.003156 · Source: PubMed

CITATIONS

36

8 authors, including:

\section{Caroline Lecaplain}

The University of Arizona

63 PUBLICATIONS 589 CITATIONS

SEE PROFILE

Eric Cormier

University of Bordeaux

217 PUBLICATIONS 2,616 CITATIONS

SEE PROFILE

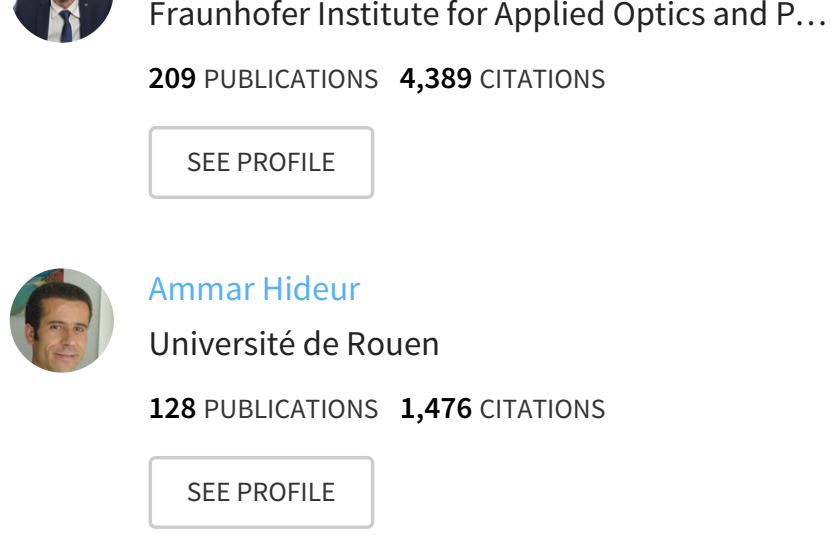

\section{Thomas Schreiber}

Fraunhofer Institute for Applied Optics and P... 209 PUBLICATIONS 4,389 CITATIONS

SEE PROFILE

Ammar Hideur

Université de Rouen

128 PUBLICATIONS 1,476 CITATIONS

SEE PROFILE

Some of the authors of this publication are also working on these related projects:

Project Matter-wave laser Interferometric Gravitation Antenna (MIGA) View project

Project High Power Fiber Laser Systems View project 


\title{
High-energy femtosecond photonic crystal fiber laser
}

\author{
Caroline Lecaplain, ${ }^{1, *}$ Bülend Ortaç, ${ }^{2,4}$ Guillaume Machinet, ${ }^{3}$ Johan Boullet, ${ }^{3}$ Martin Baumgartl, ${ }^{4}$ \\ Thomas Schreiber, ${ }^{5}$ Eric Cormier, ${ }^{3}$ and Ammar Hideur ${ }^{1}$ \\ ${ }^{1}$ CNRS UMR 6614 CORIA, Université de Rouen, Avenue de l'Université, BP 12, 76801 Saint Etienne du Rouvray, France \\ ${ }^{2}$ UNAM-Institute of Materials Science and Nanotechnology, Bilkent University, 06800 Bilkent, Ankara, Turkey \\ ${ }^{3}$ CELIA, Université Bordeaux 1, 351 Cours de la Libération F-33405 Talence, France \\ ${ }^{4}$ Institute of Applied Physics, Albert-Einstein-Strasse 15, D-07745 Jena, Germany \\ ${ }^{5}$ Institute for Applied Optics and Precision Engineering, Albert-Einstein-Strasse 7, D-07745 Jena, Germany \\ *Corresponding author: lecaplain@coria.fr
}

Received July 23, 2010; revised August 27, 2010; accepted August 30, 2010;

posted August 31, 2010 (Doc. ID 132159); published September 20, 2010

\begin{abstract}
We report the generation of high-energy high-peak power pulses in an all-normal dispersion fiber laser featuring large-mode-area photonic crystal fibers. The self-starting chirped-pulse fiber oscillator delivers $11 \mathrm{~W}$ of average power at $15.5 \mathrm{MHz}$ repetition rate, resulting in $710 \mathrm{~nJ}$ of pulse energy. The output pulses are dechirped outside the cavity from $7 \mathrm{ps}$ to nearly transform-limited duration of $300 \mathrm{fs}$, leading to pulse peak powers as high as 1.9 MW. Numerical simulations reveal that pulse shaping is dominated by the amplitude modulation and spectral filtering provided by a resonant semiconductor saturable absorber. @ 2010 Optical Society of America OCIS codes: $320.7090,320.5540,140.7090$.
\end{abstract}

High-power femtosecond laser sources are versatile tools for numerous applications ranging from material processing on a submicrometer scale to high-field physics. Developing compact and robust oscillators of high-energy femtosecond pulses has therefore generated strong research interest during the past decade, leading to significant advances in the field. In particular, impressive performance levels, with microjoule energies and up to hundreds of watts average powers, have been achieved with thin-disk lasers based on Yb-doped crystals [1, [2]. Another promising solution for energy scaling in mode-locked oscillators is rare-earth-doped fibers. Fiberbased sources exhibit very high gain per pass, excellent thermo-optical properties, and high mechanical stability, making them very suitable for high-power applications. The fundamental challenge for ultrafast fiber lasers relies on the control of excessive nonlinearity, which hinders a self-consistent pulse evolution at high energies. To some extent, the pulse energy can be scaled by increasing the amount of net positive cavity dispersion, which tends to scale down the peak power inside the fiber core by stretching the pulse during its propagation. This is the principle underlying stretched-pulse [3] and similariton lasers [四]. More recently, new routes for energy scaling of mode-locked fiber oscillators have been opened with the development of all-normal dispersion fiber (ANDF) lasers [5-8]. To achieve self-consistent pulse evolution, such lasers need a strong pulse shaping mechanism, which could be provided by a passive spectral filter (SF) [6-8] or by combination of self-amplitude modulation with gain filtering [5]. Pulse energies of more than $20 \mathrm{~nJ}$ are routinely generated in ANDF lasers based on standard single-mode fibers [7,8]. Moreover, the employment of low-nonlinearity large-mode-area photonic crystal fibers (PCFs) enables significant power scaling. This has been demonstrated recently in ANDF laser configurations using different pulse shaping mechanisms [9-14]. Notably, the extension of this approach to photonic crystal rods has produced subpicosecond (700 fs) pulses with microjoule energy levels [14].
In this Letter, we report the generation of femtosecond pulses from a highly normal dispersion fiber laser featuring a large-mode-area PCF and an Yb-doped rod-type fiber. The use of a long passive fiber allows controlling the total net cavity dispersion and the accumulated nonlinear phase, leading to the generation of linearly chirped pulses. The laser performances reach more than $11 \mathrm{~W}$ of average power at a $15.5 \mathrm{MHz}$ repetition rate, corresponding to $710 \mathrm{~nJ}$ pulses. The output pulses are extracavity dechirped down to $300 \mathrm{fs}$ transform-limited duration with 1.9 MW peak power. To the best of our knowledge, this is the highest peak power ever reached by a modelocked fiber laser.

The passively mode-locked fiber laser is constructed in a sigma configuration around a polarization-sensitive optical isolator (Fig. (1)). The gain fiber consists in a 95-cmlong $\mathrm{Yb}$-doped PCF, with a mode-field diameter of $70 \mu \mathrm{m}$ and effective NA $\sim 0.01$. The inner cladding has a diameter of $200 \mu \mathrm{m}$ and a very large effective NA $>0.7$. The fiber is cladding pumped with a fiber-coupled laser diode emitting at $976 \mathrm{~nm}$. The fiber ends are polished at an angle of $5^{\circ}$ to eliminate parasitic reflections. The rejection port of the polarizing beam splitter serves as a variable output. Polarization controllers are used to minimize cavity losses and adjust the output coupling ratio. The positive net cavity dispersion is increased by adding a 6-mlong passive PCF fiber with a mode-field diameter of $\sim 20 \mu \mathrm{m}$. Insertion of the passive fiber after the output coupling allows controlling the accumulated nonlinear

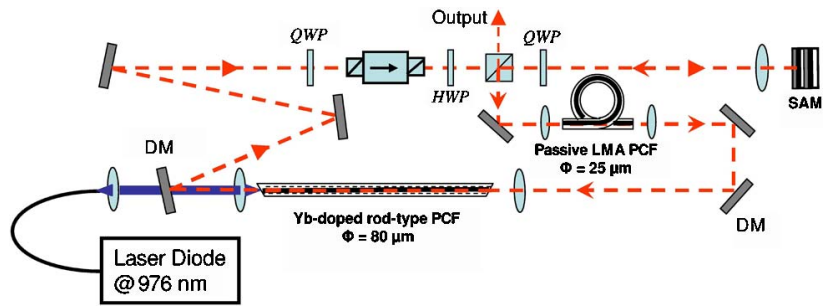

Fig. 1. (Color online) Experimental setup of the mode-locked Yb-doped PCF laser: DM, dichroic mirror; HWP, QWP, half- and quarter-wave plate. 
phase. The total cavity dispersion is about $+0.138 \mathrm{ps}^{2}$. The cavity includes a free-space section of $\sim 9 \mathrm{~m}$. Passive mode locking is achieved using fast saturable absorber mirrors (SAMs) introduced in the sigma branch (Fig. (1). The SAMs are based on InGaAs multiquantum well structures grown on a multilayer GaAs/AlAs Bragg mirror. Two different structures with a resonant and an antiresonant design have been used in this work. Both structures exhibit a low intensity absorption of $\sim 30 \%$, a modulation depth of $\sim 20 \%$, and $\sim 500$ fs relaxation time. The antiresonant SAM presents a saturation fluence of $120 \mu \mathrm{J} / \mathrm{cm}^{2}$, which is twice that of the resonant SAM. The nearly resonant design ensures a narrow absorption bandwidth of $\sim 20 \mathrm{~nm}$ FWHM.

For both structures, by optimizing the saturation criteria on the SAM using an adequate focusing lens, a self-starting mode-locking regime is obtained for a pump power of $\sim 20 \mathrm{~W}$. The laser produces a stable single-pulse train at a $15.5 \mathrm{MHz}$ repetition rate, which is monitored with a fast photodiode and a $8 \mathrm{GHz}$ sampling oscilloscope. A long scan range ( $>150 \mathrm{ps)}$ autocorrelator is used for single-pulse operation checking and pulse characterization. The single-pulse regime remains stable up to the maximal pump power of $\sim 40 \mathrm{~W}$. The average output power is then $11 \mathrm{~W}$, which corresponds to $\sim 710 \mathrm{~nJ}$. The reported results correspond to a roughly estimated output coupling ratio of $\sim 75 \%$. For higher values, the modelocking operation became unstable, suggesting that nonlinear polarization evolution (NPE) contributes to pulse shaping. The typical results obtained with the antiresonant SAM are summarized in Fig. 2 The laser generates highly chirped 13.5 ps pulses (assuming a Gaussian shape) with a spectral width (FWHM) of $10 \mathrm{~nm}$ around $1030 \mathrm{~nm}$. The optical spectrum exhibits a steep edged shape with a parabolic top, which is typical of an ANDF laser with low self-phase modulation (SPM) action. Using a transmission grating pair providing $-3.2 \mathrm{ps}^{2}$, the output pulses are extracavity dechirped to 500 fs duration [Fig. 2(b)]. This corresponds to $\sim 1.4$ times the transformlimited duration of $350 \mathrm{fs}$. The typical output spectrum obtained with the resonant SAM always exhibits the typical steep edged shape with a parabolic top [Fig. 3(a)]. It is centered at $1033 \mathrm{~nm}$ with a spectral width of $11.6 \mathrm{~nm}$. The output autocorrelation trace is best fitted assuming a Gaussian pulse of $7 \mathrm{ps}$ width [Fig. 3(b)]. The output pulses are dechirped externally to a nearly transformlimited duration of $300 \mathrm{fs}$ [Fig. 3(c)] The optimum pulse compression is achieved for $-1 \mathrm{ps}^{2}$ total net dispersion.
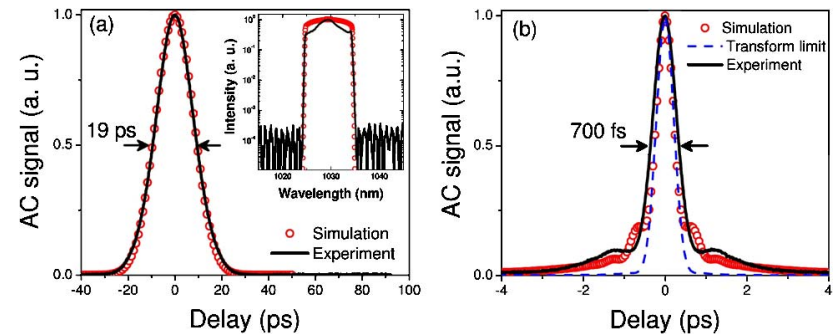

Fig. 2. (Color online) Outputs of the antiresonant SAM-based laser (solid curves): pulse autocorrelation (a) before and (b) after external compression. Inset, corresponding optical spectrum. Also shown are the numerical results calculated for $1 \mu \mathrm{J}$ intracavity pulse energy (dots).
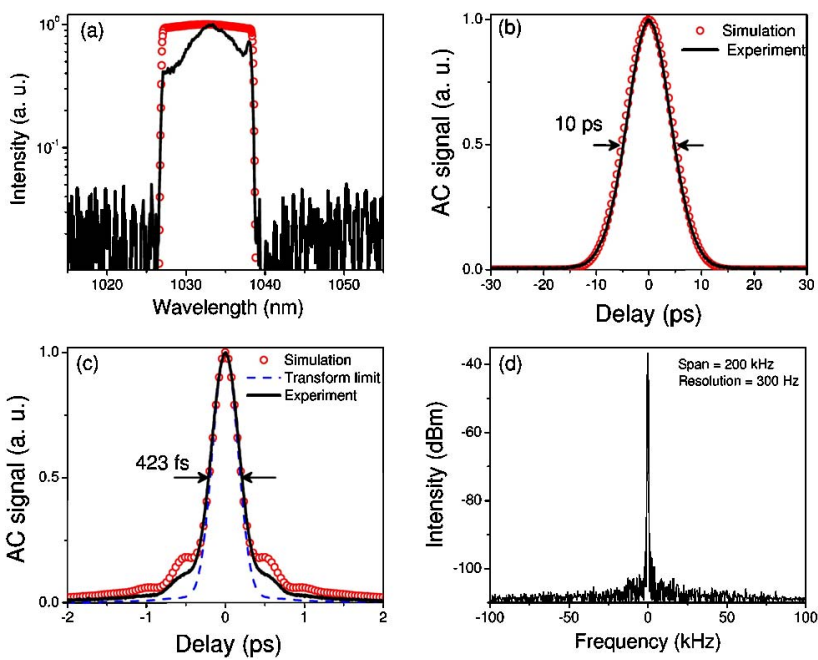

Fig. 3. (Color online) Outputs of the resonant SAM-based laser (solid curves): (a) optical spectrum, pulse autocorrelation (b) before and (c) after external compression, and (d) rf spectrum. Also shown are the numerical results calculated for $1 \mu \mathrm{J}$ intracavity pulse energy (dots).

The extracavity compressor introduces $20 \%$ additional losses; hence, the dechirped pulse energy is $\sim 570 \mathrm{~nJ}$. This corresponds to $\sim 1.9$ MW peak power. Mode locking was sustained over several hours of operation. The amplitude noise level, measured by a rf analyzer [Fig. 3(d)], is lower than $0.4 \%$, which confirms the good stability of the mode-locked operation.

To understand the operation of the reported laser, numerical simulations were performed using the arrange-

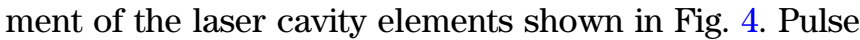
propagation along the gain fiber is described by the extended nonlinear Schrödinger equation, which includes the effects of dispersion, Kerr nonlinearity, and saturated gain with a finite bandwidth of $40 \mathrm{~nm}[[7,[15]$. Suggesting that the NPE mechanism plays a key role in pulse shaping, an ideal saturable absorber with monotonically increasing transmission is introduced just after the gain fiber [7,[15]. Absorption of the SAM is described by the
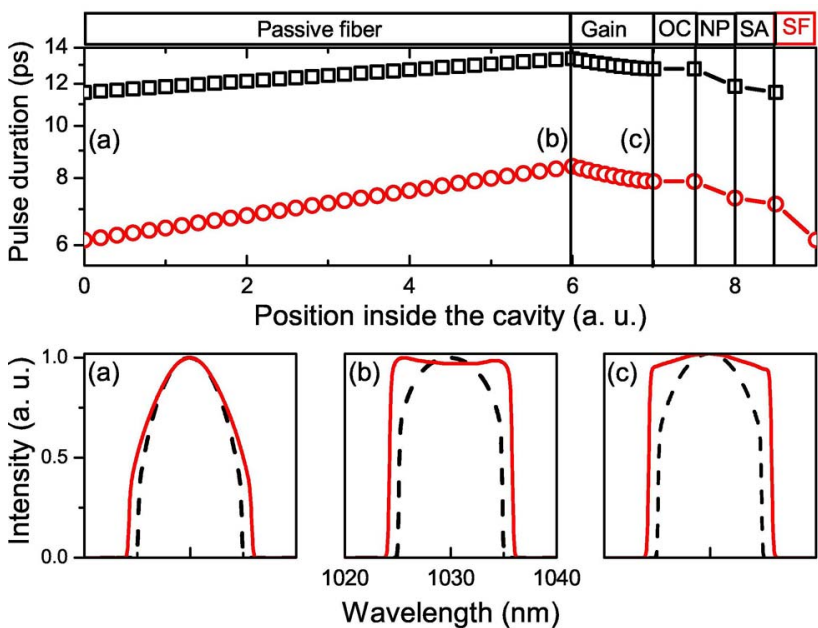

Fig. 4. (Color online) Top, temporal pulse evolution with (open circles) and without (open squares) SF. Bottom, optical spectra calculated at various locations with (solid curves) and without (dashed curves) SF: OC, output coupler; NP, NPE port; SA, saturable absorber. 
rate equation model with a relaxation time of $500 \mathrm{fs}$ [13]. To consider the finite bandwidth of the resonant SAM, a passive SF with $20 \mathrm{~nm}$ width is introduced in the numerical model. The simulation starts from quantum noise, and after convergence, the intracavity pulse evolution and the output pulse characteristics are calculated.

For both configurations, with and without the SF, stable pulse solutions do exist for intracavity energies varying from $\sim 100 \mathrm{~nJ}$ to several microjoules. As expected, the numerical results obtained without the SF are in good agreement with the antiresonant SAM experiments (Fig. 2). The little discrepancy observed on the optical spectrum could be attributed to the spectral discrimination through NPE, which is not accurately described by our scalar model. The simulation shows that pulse shaping is dominated by self-amplitude modulation provided by NPE and the SAM (Fig. 团). We note that gain filtering contributes significantly to directly shorten the pulse in the time domain. This behavior is governed by the high net cavity dispersion. Indeed, insertion of a long passive fiber enables temporal pulse broadening just behind the gain fiber. This contributes to increase the amplitude modulation induced by gain filtering, which acts on highly chirped pulses. Moreover, the temporal evolution along the cavity resembles that of the self-similar laser [四], except that the dispersion delay line is replaced by the combined actions of NPE and the SAM. Indeed, the monotonic evolution along the passive fiber indicates that the cumulated frequency chirp is mainly linear. The negligible SPM endured along the gain fiber allows maintaining the linear chirp, which is partially removed by the SAM and NPE actions. The parabolic-top spectrum obtained all along the cavity is an additional signature of self-similar pulse evolution (Fig. (1)). The tendency for self-similar pulse propagation could be related to the low nonlinear phase accumulated along the cavity, which is $\sim 1.8 \pi$, as already demonstrated in [7].

Numerical simulations with the addition of the SF inside the cavity are also in good agreement with the resonant SAM experiments (Fig. B). Shorter pulses with higher peak powers and slightly broader spectra are generated due to the additional pulse shaping action induced by the SF. Indeed, the corresponding accumulated nonlinear phase is $\sim 2.2 \pi$. Surprisingly, beside a slight increase of the temporal lengthening, the temporal pulse evolution remains basically the same as without the SF (Fig. (1). The quasi-linear chirp is also preserved all along the cavity, and the output pulses are extracavity dechirped to transform-limited duration.

In conclusion, we have presented a highly normal dispersion $\mathrm{Yb}$-doped fiber laser based on a rod-type PCF. The laser delivers $11 \mathrm{~W}$ at $15.5 \mathrm{MHz}$ repetition rate. The $710 \mathrm{~nJ}$ pulses can be dechirped to $300 \mathrm{fs}$. Peak powers as high as $1.9 \mathrm{MW}$ can be reached with this laser, revealing the scalability of the concept to approach the highest solid-state laser performances. Numerical simulations reveal that pulse evolution is very close to the similariton laser and pulse shaping is dominated by the amplitude modulation provided by the SAM and NPE in combination with passive spectral filtering.

This work is supported by the Inter Carnot-Fraunhofer program under project APUS and the French Agency for Research under project OFFEMET. We acknowledge support from the Conseil Régional de Haute Normandie.

\section{References}

1. J. H. Neuhaus, D. Bauer, J. Zhang, A. Killi, J. Kleinbauer, M. Kumkar, S. Weiler, M. Guina, D. H. Sutter, and T. Dekorsy, Opt. Express 16, 20530 (2008).

2. C. Baer, C. Kränkel, C. Saraceno, O. Heckl, M. Golling, R. Peters, K. Petermann, T. Südmeyer, G. Huber, and U. Keller Opt. Lett. 35, 2302 (2010).

3. K. Tamura, E. P. Ippen, H. A. Haus, and L. E. Nelson, Opt. Lett. 18, 1080 (1993).

4. F. Ö. Ilday, J. R. Buckley, W. G. Clark, and F. W. Wise, Phys. Rev. Lett. 92, 213902 (2004).

5. L. M. Zhao, D. Y. Tang, and J. Wu, Opt. Lett. 31, 1788 (2006).

6. A. Chong, J. Buckley, W. Renninger, and F. Wise, Opt. Express 14, 10095 (2006).

7. A. Chong, W. H. Renninger, and F. W. Wise, J. Opt. Soc. Am. B 25, 140 (2008).

8. K. Kieu, W. H. Renninger, A. Chong, and F. W. Wise, Opt. Lett. 34, 593 (2009).

9. C. Lecaplain, C. Chédot, A. Hideur, B. Ortaç, and J. Limpert, Opt. Lett. 32, 2738 (2007).

10. B. Ortaç, O. Schmidt, T. Schreiber, J. Limpert, A. Tünnermann, and A. Hideur, Opt. Express 15, 10725 (2007).

11. C. Lecaplain, B. Ortaç, and A. Hideur, Opt. Lett. 34, 3731 (2009).

12. S. Lefrançois, K. Kieu, J. Deng, J. D. Kafka, and F. W. Wise, Opt. Lett. 35, 1569 (2010).

13. M. Baumgartl, B. Ortaç, C. Lecaplain, A. Hideur, J. Limpert, and A. Tünnermann, Opt. Lett. 35, 2311 (2010).

14. B. Ortaç, M. Baumgartl, J. Limpert, and A. Tünnermann, Opt. Lett. 34, 1585 (2009).

15. T. Schreiber, B. Ortaç, J. Limpert, and A. Tünnermann, Opt. Express 15, 8252 (2007). 